\title{
Reinstatement after counterconditioning
}

\author{
DOUGLAS C. BROOKS, BETH HALE, JAMES B. NELSON, and MARK E. BOUTON \\ University of Vermont, Burlington, Vermont
}

\begin{abstract}
Reinstatement after counterconditioning was examined in three experiments with rats. The rats received CS-shock pairings in Phase 1 and then CS-food pairings in Phase 2. When unsignaled shock was presented after appetitive conditioning, fear performance to the CS replaced food performance. This reinstatement effect depended on initial pairings of the CS and shock in Phase 1. It also depended on shock exposure occurring in the test context. The results parallel previous data obtained after extinction. Counterconditioning and extinction yield several parallel effects (spontaneous recovery, renewal, and now reinstatement) which suggest that Phase 2 does not destroy the learning acquired in Phase 1.
\end{abstract}

Performance to a conditioned stimulus (CS) can recover following extinction. Recovery of performance after extinction suggests that extinction does not result in unlearning; that is, information acquired during conditioning is not destroyed by a procedure in which the CS is repeatedly presented without the unconditioned stimulus (US; Bouton, 1991, 1993). One recovery effect is spontaneous recovery, the return of the conditioned response (CR) that occurs when time passes following extinction (e.g., Brooks \& Bouton, 1993; Pavlov, 1927; Rescorla \& Cunningham, 1978; Robbins, 1990; Thomas \& Sherman, 1986). Another is the renewal effect, the return of the CR that occurs when the background context is switched following extinction (e.g., Archer, Sjödén, Nilsson, \& Carter, 1979; Bouton \& Bolles, 1979; Bouton \& King, 1983; Bouton \& Peck, 1989; Bouton \& Ricker, 1994; Bouton \& Swartzentruber, 1989; Brooks \& Bouton, 1994). A third example is reinstatement, in which the extinguished CR returns when the CS is tested after exposures to the US alone (e.g., Bouton, 1984; Bouton \& Bolles, 1979; Bouton \& King, 1983; Bouton \& Peck, 1989; Rescorla \& Cunningham, 1978; Rescorla \& Heth, 1975; cf. Baker, Steinwald, \& Bouton, 1991).

Bouton (1993) has recently noted parallels between extinction and counterconditioning, another procedure in which the $\mathrm{CS}$ is associated with a different event after conditioning with a particular US. In counterconditioning, the CS is paired with a second, qualitatively different US in the second phase. For example, aversive-appetitive transfer involves pairing the CS with an aversive US (e.g., shock) in Phase 1 and an appetitive US (e.g., food or water) in Phase 2. The second phase establishes a CR (e.g., magazine entry) different from that conditioned in Phase 1 (freezing). (In appetitive-aversive transfer, each phase in-

This research was supported by Grants BNS 89-08535 and IBN $92-$ 09454 from the National Science Foundation to M.E.B. Correspondence concerning this article should be sent to Mark E. Bouton, Department of Psychology, University of Vermont, Burlington, VT 05405. D. C. Brooks can now be reached at St. Michael's College, Psychology Department, Winooski Park, Colchester, VT 05439. volves the alternate US and CR.) As in extinction, the information acquired during Phase 1 does not appear to be destroyed by counterconditioning in Phase 2 (Bouton, 1993). Spontaneous recovery of Phase 1 performance has been demonstrated when time is allowed to pass following Phase 2 (Bouton \& Peck, 1992). And renewal of Phase 1 performance has been demonstrated following a switch to the Phase 1 context after Phases 1 and 2 take place in different contexts (Peck \& Bouton, 1990).

We are not aware of any research that has investigated reinstatement after counterconditioning. A demonstration of this effect would further support the similarity between extinction and counterconditioning. This was the objective of the present experiments. We used an aversive-appetitive transfer paradigm in which a CS was paired with a shock US in Phase 1 and then a food US in Phase 2. Experiment 1 established a new method that yields representative results. We then used the method to ask whether exposure to Phase 1 shock USs would reinstate aversive conditioning performance after Phase 2. The results from Experiments 2 and 3 suggest that Phase 1 shock USs do reinstate Phase 1 aversive conditioning performance after appetitive counterconditioning. Reinstatement depended on CS-US pairings in Phase 1 (Experiment 2). It also depended on presenting the reinstating shock USs in the context in which testing was to occur (Experiment 3; cf. Bouton, 1984; Bouton \& Bolles, 1979; Bouton \& King, 1983; Bouton \& Peck, 1989).

\section{EXPERIMEN'T 1}

In the first experiment, aversive-appetitive transfer was examined using a new method. CSs and USs were superimposed on a baseline magazine-entry response that was maintained by food pellets delivered on a variable time (VT) schedule. Pairings of the CS and shock in Phase 1 should suppress the baseline response during the CS. Subsequent pairings of the $\mathrm{CS}$ and food in Phase 2 should reduce this suppression and then enhance the baseline response during the CS (e.g., DeCola \& Rosellini, 1990).

The design is presented in Table 1. Group AV (aversive conditioning) received CS -shock pairings in Phase 1, then CS-food pairings in Phase 2. Group NAV (no aversive 
Table 1

Design of Experiments

\begin{tabular}{lllll}
\hline \multicolumn{5}{c}{ Phase } \\
Group & Aversive & Appetitive & Reinstatement & Test \\
\cline { 3 - 4 } & \multicolumn{5}{c}{ Experiment 1 } \\
AV & CS-SH & CS-F & \\
NAV & - & CS-F & \\
EU & CS/SH & CS/F & \\
& & Experiment 2 & & \\
R & CS-SH & CS-F & SH & CS \\
NR & CS-SH & CS-F & - & CS \\
EU & CS/SH & CS/F & SH & CS \\
& & Experiment 3 & & \\
Same & A:CS-SH & A:CS-F & A:SH & A:CS \\
Diff & A:CS-SH & A:CS-F & B:SH & A:CS \\
\hline
\end{tabular}

Note-SH, shock, F, food; an en dash (-) designates pairings of the CS and US (either shock or food); a slash (/) designates presentation of the CS and US in an explicitly unpaired manner; em dashes (-) indicate sessions in which no CSs or USs occurred.

conditioning) received only Phase $2 \mathrm{CS}$-food pairings. In each phase, Group EU (explicitly unpaired) received the same CSs and USs as did Group AV, but they were presented in an explicitly unpaired manner. This unpaired procedure should not yield conditioning in either phase (see Bromage \& Scavio, 1978; Peck \& Bouton, 1990; Scavio, 1974). In Phase 1 , aversive conditioning should produce greater baseline suppression in Group AV than in Group EU. In Phase 2, appetitive conditioning should produce greater baseline responding in Groups NAV and AV than in Group EU. Since aversive conditioning typically interferes with the acquisition of appetitive conditioning in Phase 2 (e.g., Bouton \& Peck, 1992; Bromage \& Scavio, 1978; Kaye, Preston, Szabo, Druiff, \& Mackintosh, 1987; Peck \& Bouton, 1990; Scavio, 1974), if the present method yields representative results, then Group AV should acquire appetitive conditioning more slowly than Group NAV.

\section{Method}

\section{Subjects}

The subjects were 24 experimentally naive female Wistar rats bred at the University of Vermont. They were approximately 100 days old at the start of the experiment and were housed individually in standard stainless steel cages in a room maintained on a 18:6-h light-dark cycle. The experiment was conducted on consecutive days during the light portion of the cycle. The rats were food deprived and kept at $80 \%$ of their initial body weights throughout the experiment.

\section{Apparatus}

The experiment was conducted in two sets of four Skinner boxes located in different rooms of the laboratory. Each box in one set measured $26 \times 25 \times 19 \mathrm{~cm}$. The back and one side wall were made of aluminum. The other walls and the ceiling were made of clear acrylic plastic. The floor of each box was constructed of tubular steel bars $16 \mathrm{~mm}$ in diameter and spaced $3.2 \mathrm{~cm}$ from center to center. The bars were perpendicular to the front wall where a recessed stainless steel food cup was centered. Each cup was positioned $3 \mathrm{~cm}$ above the floor and was accessed through a 6-cm-square opening. Inactive operant levers were positioned $6 \mathrm{~cm}$ above the floor and $2 \mathrm{~cm}$ to the right of the food cup. The animals entered the boxes through a door
}

in the right side wall. A distinctive olfactory cue was provided by a dish containing $10 \mathrm{ml}$ of Heinz white vinegar (H. J. Heinz Co.. Pittsburgh, PA), which was replenished daily and placed outside the Skinner box, below the food cup.

Each box in the second set of boxes measured $24 \times 22 \times 18 \mathrm{~cm}$. The back wall was aluminum, the remaining walls and ceiling were made of clear acrylic plastic. Black vertical stripes, $2 \mathrm{~cm}$ in width and spaced $2.5 \mathrm{~cm}$ apart, were on the front, back, and left side walls. The floor was constructed of stainless steel bars $3 \mathrm{~mm}$ in diameter and spaced $1.5 \mathrm{~cm}$ from center to center. The bars were parallel to the front wall. A recessed stainless steel food cup was centered on the front wall and positioned $3 \mathrm{~cm}$ above the floor; it was accessed through a $6-\mathrm{cm}$-square opening. Inactive operant levers were positioned $6 \mathrm{~cm}$ above the floor and $6 \mathrm{~cm}$ to the left of the food cup. The animals cntered the boxes through the ceiling. The olfactory cue was approximately 1 g of Vicks Vaporub (Richardson-Vicks, Inc., Shelton, CT) which was replenished daily and placed outside the Skinner box. below the food cup.

The CS was a $30-\mathrm{sec}, 3000-\mathrm{Hz}$ tone ( $80 \mathrm{~dB}$ re $20 \mu \mathrm{N} / \mathrm{M}^{2}[\mathrm{~A}]$ ) provided by a single generator wired to identical speakers positioned $25 \mathrm{~cm}$ above the floor of each box. Illumination was provided by two $7.5-\mathrm{W}$ white incandescent bulbs mounted $25 \mathrm{~cm}$ above the floors During aversive conditioning, the US was a $0.5-\mathrm{sec}, 0.6-\mathrm{mA}$ footshock provided by Grason-Stadler shock sources and scramblers: during appetitive conditioning, the US was three $45-\mathrm{mg}$ food pellets (Noyes, Traditional Formula. P. J. Noyes, Lancaster, NH) delivered $0.2 \mathrm{sec}$ apart. Magazine entries were detected by photocells mounted within the magazines just behind the plane of the wall of the Skinner boxes.

\section{Procedure}

The experiment was run on consecutive days; each rat received one session per day and was run in the same box throughout the experiment.

Baseline training. During three daily baseline training sessions. each rat was trained to enter the food magazine. This was accomplished by programming delivery of individual food pellets on a VT 120-sec schedule (range of 80-150 sec). Each session was $60 \mathrm{~min}$ in duration and involved 30 pellet deliveries. In this and subsequent experiments, CS and US presentations were later superimposed on the VT schedule; however, baseline pellet deliveries were always programmed so that no pellet was delivered within $60 \mathrm{sec}$ of any CS or any shock or three-pellet food US.

Aversive conditioning. Prior to the start of this phase, the rats were assigned randomly to one of three groups $(n=8)$, with the restriction that the groups had demonstrated similar magazine-entry responding during baseline training. During the next four sessions, Group AV received aversive conditioning. Each 60 -min session involved two presentations of the CS terminating with the shock US. CSs occurred at Minutes 17 and 54. Group EU received the same number of CSs and shock USs in an explicitly unpaired manner; the minimum CS -US interval was $9 \mathrm{~min}$. Timing of the CS within the session was the same for both groups. Group NAV received 60-min baseline training sessions during this phase.

Appetitive conditioning. In each of the next three sessions, Groups AV and NAV received appetitive conditioning. Each $70-\mathrm{min}$ session involved 12 presentations of the CS terminating with the three-pellet food US. The first CS occurred at Minute 2; subsequent CSs occurred with a mean ITI of $5.5 \mathrm{~min}$ and a range of 3-11 min. Group EU received the same number of CSs and food USs presented in an explicitly unpaired manner; the minimum CS-US interval was $4 \mathrm{~min}$. CS timing was the same for all groups.

Data collection and analysis. The number of magazine entries made during the $30-\mathrm{sec} C S$ was counted along with the number made during the 30-sec period immediately prior to CS onset (the pre-CS period). On each trial, an elevation score was calculated by subtracting the number of entries made during the pre-CS period from the number made during the CS. Elevation scores were analyzed 
using analyses of variance (ANOVAs); corresponding pre-CS scores were analyzed in separate ANOVAs. Planned comparisons were conducted using the standard methods discussed by Howell (1987, pp. 330-337, 431-441). The rejection criterion was always $p<.05$.

\section{Results and Discussion}

There were no differences in pre-CS responding between the groups at any point in the experiment (all $p s>.10$ ). Aversive and appetitive conditioning were both demonstrated with the present method. Aversive conditioning in Phase 1 also interfered with the acquisition of appetitive performance in Phase 2.

Figure 1 presents mean elevation scores during the 9 four-trial blocks of the appetitive conditioning phase. The figure suggests that at the start of the phase, Group AV suppressed responding to the CS compared to Group EU; Phase 1 CS-shock pairings had resulted in conditioned. suppression of the baseline. Over the course of Phase 2, Group AV then acquired appetitive performance, but did so less rapidly than Group NAV. By the end of the phase, however, Groups NAV and AV demonstrated comparable appetitive performance.

A group $\times$ block ANOVA confirmed these observations. There was a significant effect of group $[F(2,21)=$ $8.35]$. There was also an effect of block $[F(8,168)=2.98]$, indicating an overall increase in responding during the phase. The interaction between these factors was also significant $[F(16,168)=3.60]$. Planned comparisons on the first block revealed that responding in Group AV was suppressed compared to both Groups NAV and EU [smallest $F(1,21)=9.56]$. Groups NAV and EU did not differ on this block $[F(1,21)<1]$. Phase 1 aversive conditioning clearly produced conditioned suppression in Group AV.

On Blocks 4-6, Group NAV demonstrated appetitive conditioning performance; responding in this group was greater than that in Group EU [smallest $F(1,21)=4.48$ ]. However, Group AV did not differ from Group EU on these blocks [largest $F(1,21)=2.38$ ], indicating that Group AV did not yet demonstrate appetitive conditioning. On the

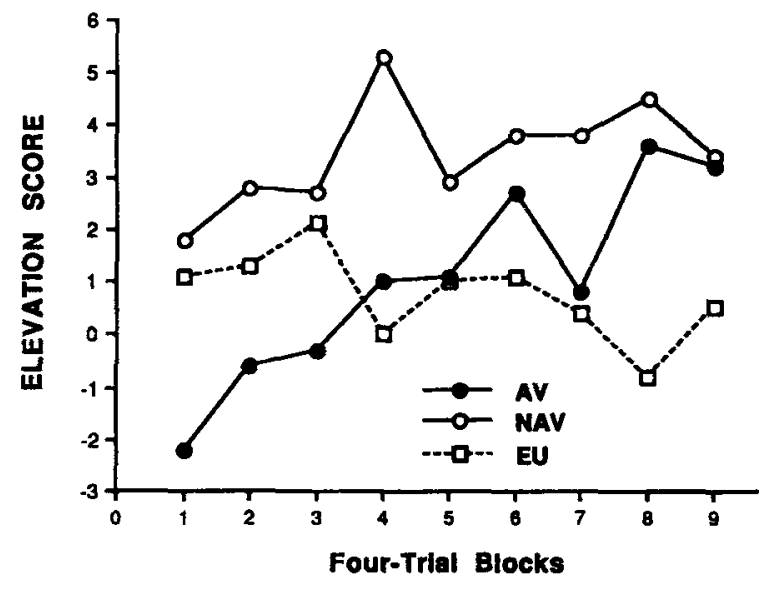

Figure 1. Mean elevation scores during the 9 four-trial blocks of Phase 2 appetitive conditioning for Groups AV, NAV, and EU in Experiment 1. final two blocks, however, both Group AV and Group NAV demonstrated appetitive conditioning; responding was greater in each group than in Group EU [smallest $F(1,21)=$ 6.26]. Groups AV and NAV did not differ on either block $[F \mathrm{~s}(1,21)<1]$.

The results suggest that both aversive and appetitive conditioning performance were demonstrated by using the present procedure. They are consistent with data from previous studies in suggesting that aversive conditioning interferes proactively with the acquisition of appetitive conditioning performance (e.g., Bouton \& Peck, 1992; Bromage \& Scavio, 1978; Kaye et al., 1987; Peck \& Bouton, 1990 ; Scavio, 1974). The present method is therefore suitable for investigating reinstatement in aversive-appetitive transfer.

\section{EXPERIMENT 2}

In Experiment 2, we began to investigate the reinstatement of Phase 1 aversive-conditioning performance following appetitive counterconditioning. The design is presented in Table 1. In Phase 1, Groups R (reinstatement) and NR (no reinstatement) received $C S$-shock pairings; in Phase 2 , they received CS-food pairings. In each phase, Group EU received the CS and US in an explicitly unpaired manner which again should not yield conditioning. Following Phase 2, Groups $\mathrm{R}$ and EU received exposure to Phase 1 shock USs while Group NR received no shocks. The groups were then tested for responding to the CS. If Phase 1 shock USs were to reinstate aversive conditioning performance following appetitive conditioning, responding on the test should be resuppressed in Group R. Since reinstatement after extinction depends on an original CS-shock association (e.g., Bouton \& Bolles, 1979), we expected little change in performance in Group EU. Group NR did not receive exposure to Phase 1 USs and should continue to demonstrate appetitive conditioning.

\section{Method}

\section{Subjects}

The subjects were 24 male and 24 female Wistar rats bred at the University of Vermont. They were approximately 100-120 days old at the start of the experiment. The experiment was run in two replications. Rats in the first replication $(n=24)$ were experimentally naive. Rats in the second replication $(n=24)$ had been run in a previous study in a separate apparatus that involved exposure to food pellets and to a CS that differed from the one used here. Rats from the previous experimental conditions were orthogonally assigned to groups in the present experiment. The housing and maintenance conditions were the same as in Experiment 1

\footnotetext{
Apparatus

In the first replication, the apparatus was the same as that used in Experiment 1. The second replication employed two other counterbalanced sets of four Skinner boxes. Each of these boxes measured $23 \times 13 \times 11 \mathrm{~cm}$, was made of clear acrylic plastic, and was housed in a sound-attenuation chamber. The front wall and one side wall were transparent; the exteriors of the other walls were covered with black construction paper. The rats entered the box through the ceiling. On the right wall of each box was a stainless steel recessed food cup positioned $3 \mathrm{~cm}$ above the floor; the food cup was accessed through a $6-\mathrm{cm}$-square opening. Inactive operant levers were located
} 
at the right of the back wall, $5 \mathrm{~cm}$ above the floor. In the first set of boxes, the floor consisted of 3 -mm bars mounted parallel to the side wall and staggered so that the odd-numbered bars were mounted $6 \mathrm{~mm}$ above the even-numbered bars. Odd-numbered bars were spaced $1.6 \mathrm{~cm}$ apart (as were the even ones). The side and back walls had $1-\mathrm{cm}$ horizontal white stripes spaced $1 \mathrm{~cm}$ apart. A dish containing $10 \mathrm{ml}$ of $4 \% \mathrm{McC}$ Cormick coconut extract solution (McCormick, Hunt Valley, MD), which provided a distinctive odor, was located on the floor of the sound-attenuation chamber near the food cup. In the second set of boxes, the floor consisted of 3-mm bars (spaced $1.8 \mathrm{~cm}$ apart) mounted diagonally to the chamber walls; the rear wall was black. A dish containing $10 \mathrm{ml}$ of $2 \%$ McCormick anise extract solution provided the distinctive odor.

In the first replication, the CS was the tone used in Experiment 1. In the second replication, it was the $30-\mathrm{sec}$ presentation of a $28-\mathrm{V}$ orange jeweled light (General Electric Model 313 bulb) that flashed two times per second. The light was $1 \mathrm{~cm}$ in diameter, protruded $5 \mathrm{~mm}$ into the box, and was mounted $8 \mathrm{~cm}$ above and $4 \mathrm{~cm}$ to the right of the food cup. The USs were the same as in Experiment 1.

\section{Procedure}

The experiment was nun on consecutive days with each rat receiving one session each day in the same box throughout the experiment. Groups were equally represented in the two replications, which used identical procedures except where noted.

Pretraining. Each rat received two 50 -min baseline training sessions; 25 pellets were delivered on the VT 120 -sec schedule in each session. Rats in the second replication then received two nonreinforced preexposures to the $\mathrm{CS}$ in an extra 12-min session conducted the next day. Rats in the first replication began the aversive conditioning phase on the day following the conclusion of baseline training.

Aversive conditioning. During the next five sessions, Groups $R$ and NR $(n s=16)$ received aversive conditioning. Each 50 -min session involved three presentations of the CS terminating with the shock US. The first CS occurred at Minute 6; subsequent CSs occurred with a mean ITI of $17 \mathrm{~min}$ and a range of 11-21 min. Group EU $(n=16)$ received the same number of CSs and shock USs presented in an explicitly unpaired manner; the minimum CS-US interval was 6 min. CS timing was the same for all groups.

Appetitive conditioning. During the next phase, Groups $\mathrm{R}$ and NR received appetitive conditioning. Each session involved eight presentations of the CS terminating with the three-pellet food US. The first session lasted $60 \mathrm{~min}$. In this session, the first CS was delayed to promote baseline responding after shock presentations in Phase 1; it occurred in Minute 15. Subsequent CSs occurred with a mean ITI of $6 \mathrm{~min}$ and a range of $4-8 \mathrm{~min}$. The remaining sessions of this phase were 50 min long. First trial onset varied between Minutes 5 and 9; ITIs were the same as in the first session. Group EU received the same number of CSs and food USs presented in an explicitly unpaired manner; the minimum CS-US interval was $4 \mathrm{~min}$. CS timing was the same for all groups.

In the first replication, there were six appetitive conditioning sessions; the baseline schedule was VT 120 sec throughout the experiment. In the second replication, there were nine sessions. To minimize an increase in baseline responding that developed over sessions, the baseline schedule was increased from VT $120 \mathrm{sec}$ in Sessions 1 and 2 to VT $150 \mathrm{sec}$ in Sessions 3-6 and VT $180 \mathrm{sec}$ in Sessions 7-9.

Reinstatement treatment. In the next session, Groups R and EU received exposure to shock USs. In the first replication, six shocks were delivered in $50 \mathrm{~min}$, with the first shock occurring at Minute 8; subsequent shocks occurred with a mean ITI of 6 min. In the second replication, eight shocks were delivered in $60 \mathrm{~min}$, with the first shock occurring at Minute 11; the mean ITI was also $6 \mathrm{~min}$. Group NR received no shocks in an otherwise identical session.

Test. The final session was the reinstatement test. All groups received four nonreinforced CSs in $50 \mathrm{~min}$. The first CS occurred at Minute 12; the ITI was 12 min. Group NR had received no shocks during the previous session, and should continue to demonstrate appetitive conditioning performance comparable to that of Group EU. If shock USs reinstate Phase 1 aversive conditioning performance following appetitive conditioning, Group R's responding on the test should be suppressed relative to that of Group EU,

\section{Results and Discussion}

There were no main effects or interactions involving the replication factor (all $p \mathrm{~s}>.13$ ); for simplicity, this factor was therefore excluded from the analyses. There were no pre-CS differences among the groups (all $p s>.10$ ). Aversive and appetitive conditioning performance were again demonstrated. Most importantly, after appetitive conditioning in Phase 2, exposure to Phase 1 shock USs reinstated aversive conditioning performance.

\section{Appetitive Phase}

Figure 2 presents mean elevation scores on the first (left) and final (right) two-trial block of the appetitive conditioning phase for Groups R and NR combined (rats that always had the CS and US paired) and for Group EU. (We focused on the first and final trial blocks because each replication involved a different number of Phase 2 sessions.) The figure suggests that rats in the paired condition demonstrated aversive conditioning performance (suppressed responding) during the first block and appetitive conditioning performance (enhanced responding) during the final block. The impression was confirmed by statistical analysis. A condition (paired vs. unpaired) $\times$ block ANOVA revealed a significant effect of block $[F(1,46)=$ 19.05], and a condition $\times$ block interaction $[F(1,46)=$ $13.82]$. The effect of condition was not reliable $(F<1)$. On the first block there was a difference between conditions $[F(1,46)=33.73]$, indicating suppressed responding in the paired condition. On the final block, there was again a difference $[F(1,46)=10.89]$, now indicating enhanced responding in the paired condition. Mean elevation scores for Group EU during each block did not differ from a hypothetical population mean of zero $[t \mathrm{~s}(15)<1.13]$.

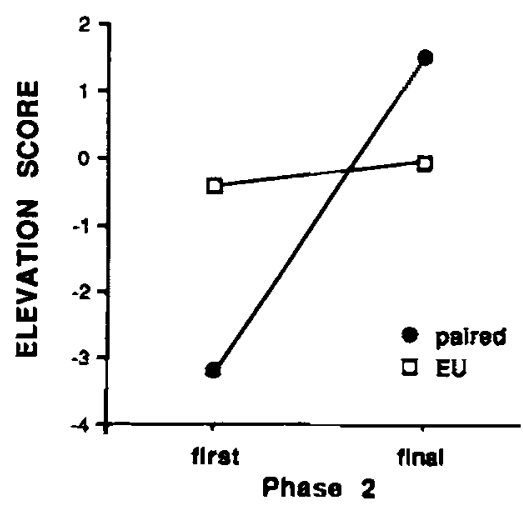

Figure 2. Mean elevation scores on the first (left) and final (right) two-trial block of Phase 2 appetitive conditioning in Experiment 2 for rats that received the $C S$ and US paired in each phase and for rats that always received the CS and US explicitly unpaired (Group EU). 


\section{Test}

Figure 3 presents mean elevation scores during the final four-trial block of appetitive conditioning (pre, left), and the four-trial block of the reinstatement test (right) for Groups R, NR, and EU. Performance on the test is of greatest interest. The figure suggests that responding in Group $R$ was resuppressed as compared with that of Group EU, while responding in Group NR remained enhanced. These impressions were confirmed by a group $\times$ block ANOVA on these data, which revealed an effect of group $[F(2,45)=4.38]$ and a significant group $\times$ block interaction $[F(2,45)=5.67]$. On the test, Group NR responded more than Group EU $[F(1,45)=4.37]$, indicating continued appetitive conditioning performance. Group R responded reliably less than Group NR $[F(1,45)=19.42]$. Furthermore, Group R's performance was suppressed as compared with that of Group EU $[F(1,45)=5.37]$, indicating reinstatement of aversive conditioning performance. Also, Group R's mean on the test was significantly below a hypothetical population mean of zero $[t(15)=$ -2.32 ], suggesting that baseline responding was suppressed in this group, as it was after Phase 1.

The results of the reinstatement test suggest that performance changes substantially when Phase 1 USs are presented noncontingently after counterconditioning. Specifically, shock exposure reinstated fear performance. The effect of shock clearly depended on initial aversive conditioning: Even though Groups $\mathrm{R}$ and EU both received shock exposure, only Group R showed reinstatement (cf. Bouton \& Bolles, 1979). Moreover, it is worth noting that shock exposure did not merely reduce appetitive performance in Group R relative to Group NR; it actually reinstated fear performance, as indicated by greater suppression in Group R than in Group EU. This result is especially interesting given the large number of Phase 2 conditioning trials involved here (48-72). Extensive experience

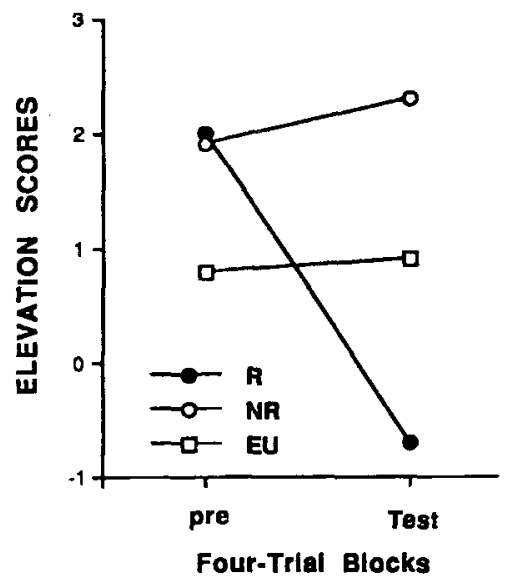

Figure 3. Mean elevation scores during the final four-trial block of Phase 2 appetitive conditioning (pre, left) and the fourtrial block of the reinstatement test (right) for Groups R, NR, and EU in Experiment 2. Groups $R$ and $N R$ received the $C S$ and $U S$ paired in each phase. Groups $R$ and $E U$ received exposure to Phase 1 shock USs; Group NR did not. with counterconditioning does not appear to destroy the CS's sensitivity to reinstatement.

\section{EXPERIMENT 3}

In the third experiment, we asked whether the reinstatement effect observed in Experiment 2 depended on exposure to Phase 1 shock USs in the test context. After extinction, reinstatement occurs in the apparatus in which exposure to Phase 1 USs recently occurred; the same US exposures in an irrelevant context produce little or no reinstatement (Bouton, 1984; Bouton \& Bolles, 1979; Bouton \& King, 1983; Bouton \& Peck, 1989; see also Baker et al., 1991). In the present experiment, we sought to evaluate whether reinstatement after counterconditioning was similarly dependent on the context of shock exposure.

Table 1 presents the experimental design. Two groups received aversive conditioning, appetitive conditioning, and testing in one context (Context A). The groups differed only with respect to the context in which they were exposed to reinstating shocks after appetitive conditioning. One group (Group Same) received shocks in the same context in which testing was to take place (Context A); the other group (Group Diff) received the shocks in a different context (Context B). If reinstatement of Phase 1 performance depends on US exposures in the test context, only Group Same should demonstrate reinstatement.

\section{Method}

\section{Subjects and Apparatus}

The subjects were 32 experimentally naive female Wistar rats obtained from Charles River Laboratories, St. Constant, Quebec. They were approximately 90 days old at the start of the experiment. The housing and maintenance conditions were the same as in the previous experiments.

The apparatus was the sets of boxes used in Experiment 1, which now provided different contexts (fully counterbalanced). The CS was the tone. USs were the same as in the previous experiments.

\section{Procedure}

The experiment was run on consecutive days, with each rat receiving one session each day. Except for the pretraining received by all rats and the reinstatement treatment given to Group Diff, each rat was run consistently in one box (Context $A$ ) throughout the experiment.

Pretraining. All rats first received $20 \mathrm{~min}$ of exposure to the boxes in which subsequent sessions were conducted. Each rat received one exposure session in Context $\mathrm{A}$ and another in Context $\mathrm{B}$; the intersession interval was approximately $120 \mathrm{~min}$. The food cups were baited with four pellets at the start of these sessions. Each rat was then given a 25-min session in each context in which it was trained to eat food pellets upon their delivery to the food cup; the intersession interval was approximately $150 \mathrm{~min}$. At the start of these sessions, food cups were baited with two pellets; the average number of USs delivered in each session was 10. All rats were then run in four consecutive daily baseline training sessions using the procedure from Experiment 2 . The first and third sessions were conducted in Context A; the second and fourth were conducted in Context B. There was no initial preexposure to the CS.

Aversive conditioning. In each of the next three sessions, each rat received aversive conditioning. Each 50 -min session involved three pairings of the CS and the shock US. The procedure was the same as that for Groups R and NR in Experiment 2. At the conclu- 
sion of this phase, 2 rats failed to demonstrate aversive conditioning; their data were not included in statistical analyses.

Appetitive conditioning. In each of the next four sessions, all rats received appetitive conditioning. Each session was $50 \mathrm{~min}$ in duration and involved eight pairings of the CS and the food US. The procedure for each session was the same as in Experiment 1 except that the baseline schedule was VT $180 \mathrm{sec}$ in the first two sessions and VT $210 \mathrm{sec}$ in the remaining two.

Reinstatement treatment. Following the end of appetitive conditioning, the rats were assigned to two groups of 15 that were matched on performance during Phase 2 . Group Same then received a session in which the shock US was presented eight times in the context that had been used throughout training (Context A). Group Diff received the same US exposure treatment in a different box (Context B), that is, a box from the alternate set of Vicks- or vinegarscented boxes (counterbalanced). For each group, the baseline schedule was VT $150 \mathrm{sec}$

Test. In the final session, all animals were run in their original boxes (Context A). Each group now received four nonreinforced CSs in $50 \mathrm{~min}$. The first CS occurred at Minute 12; the ITI was $12 \mathrm{~min}$. The baseline schedule was VT $150 \mathrm{sec}$. If the reinstatement of fear performance depends on exposure to Phase 1 shock USs in the test context, there should be a resuppression of responding on the test in Group Same but not in Group Diff.

\section{Results and Discussion}

There were no pre-CS differences between the groups at any point in the experiment (all $p s>.08$ ). Exposure to Phase 1 USs in the test context reinstated Phase 1 aversive conditioning performance in Group Same. Exposure to Phase 1 USs in a context different from the test context had no effect on performance in Group Diff.

\section{Appetitive Phase}

At left in Figure 4 are the mean elevation scores during the 16 two-trial blocks of the appetitive conditioning phase for Groups Same and Diff. This portion of the figure suggests that aversive performance at the start of Phase 2 changed to appetitive performance by the end of the phase. As in Experiment 2, we evaluated performance during the first and final two-trial block of the appetitive conditioning phase. A group $\times$ block ANOVA revealed a significant block effect $[F(1,28)=43.71]$ but no group effect or interaction $\left(F_{\mathbf{S}}<1\right)$. Both groups showed performance on the first block that was less than a hypothetical population mean of zero $[t \mathrm{~s}(14)>7.99]$ and performance on the final block that was greater than this value $[t \mathrm{~s}(14)>$ 3.79]. The unpaired control group in the previous experiment never deviated from a hypothetical population mean of zero; thus, the results for both groups suggest that aversive performance $(<0)$ was evident at the start of Phase 2 and that it had changed to appetitive performance $(>0)$ by the end of the phase.

\section{Test}

The data of greatest interest are from the reinstatement test. At right in Figure 4 are the mean elevation scores during the two two-trial blocks of the test. The figure suggests that aversive performance was reinstated in Group Same but not in Group Diff. A group $\times$ block (last Phase 2 vs. first test) ANOVA and planned comparisons confirmed these impressions. There was a significant group effect

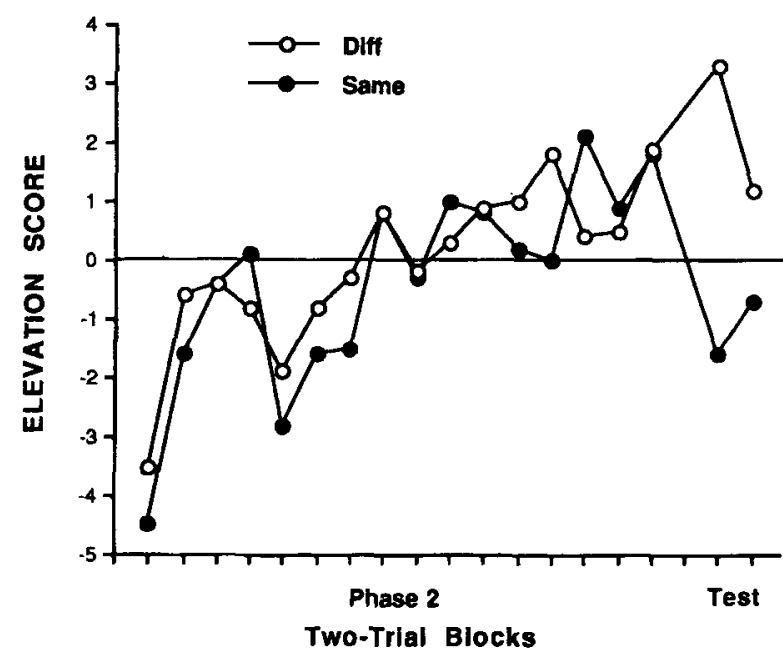

Figure 4. Mean elevation scores during the 16 two-trial blocks of Phase 2 appetitive conditioning (left) and the 2 two-trial blocks of the reinstatement test (right) for Groups Same and Diff in Experiment 3. Group Same received exposure to Phase 1 shock USs in the test context (Context $A$ ); Group Diff received the same shocks in a different context (Context B).

$[F(1,28)=11.56]$, indicating less responding in Group Same than in Group Diff. There was no block effect $[F(1,28)$ $=1.57]$. However, the group $\times$ block interaction was significant $[F(1,28)=13.46]$. Group Same's responding decreased from the end of Phase 2 to the test $[F(1,28)=$ 12.12]; Group Diff's performance did not change signif icantly $[F(1,28)<2.92, p=.10]$. On the test, Group Same was more suppressed than Group Diff $[F(1,28)=25.53]$. Group Same's performance was also significantly below a hypothetical population mean of zero $[t(14)=-3.57]$, suggesting the reinstatement of aversive conditioning performance. In contrast, Group Diff's mean on the test was significantly above a hypothetical population mean of zero $[t(14)=8.64]$, suggesting continued appetitive performance.

The results of the reinstatement test suggest that exposure to Phase 1 shock USs in the test context reinstated aversive conditioning performance while shocks presented in a different context did not. Group Same's results support those from Experiment 2 in suggesting that reinstatement of aversive performance can occur following counterconditioning with a food US. More importantly, the present results are consistent with previous ones from extinction (e.g., Bouton, 1984; Bouton \& Bolles, 1979; Bouton \& King, 1983; Bouton \& Peck, 1989; see also Baker et al., 1991) in suggesting that reinstatement after counterconditioning is especially likely when exposure to Phase 1 shock USs occurs in the test context.

\section{GENERAL DISCUSSION}

The present results can be summarized as follows. In Experiment 1, as in previous counterconditioning studies (e.g., Bouton \& Peck, 1992; Peck \& Bouton, 1990), Phase 1 conditioning interfered with the acquisition of perfor- 
mance in Phase 2. The results of Experiment 1 therefore suggest continuity between results obtained with the present method and methods described previously in the literature. More importantly, the results of Experiments 2 and 3 suggest that exposure to Phase 1 shock USs can reinstate fear performance after aversive-appetitive transfer. Reinstatement depended on initial aversive conditioning. In addition, exposure to shock USs did not merely reduce Phase 2 appetitive performance; it reinstated Phase 1 fear performance. Finally, reinstatement was most apparent when US exposures occurred in the context that was used subsequently during testing (Experiment 3 ).

The present results parallel reinstatement results previously observed after extinction (e.g., Baker et al., 1991; Bouton, 1984; Bouton \& Bolles, 1979; Bouton \& King, 1983; Bouton \& Peck, 1989; Rescorla \& Cunningham, 1978; Rescorla \& Heth, 1975). In fact, three important response-recovery effects observed after extinction have now been demonstrated after counterconditioning. In reinstatement, exposure to the Phase 1 US in the test context reinstates Phase 1 performance (and reduces Phase 2 performance); in renewal, a return to the Phase 1 context renews Phase 1 performance (and reduces Phase 2 performance; Peck \& Bouton, 1990); and in spontaneous recovery, the passage of time after Phase 2 causes recovery of Phase 1 performance (and reduces Phase 2 performance; Bouton \& Peck, 1992). Each effect illustrates an important similarity between extinction and counterconditioning: Presenting the CS alone or pairing it with a second US does not destroy the learning acquired in Phase 1. In addition, performance after either treatment is affected by manipulations of context or time.

A memory-retrieval view can integrate the range of findings (Bouton, 1991, 1993). CS-shock pairings in Phase 1 may result in the storage of a memory representation involving the CS (e.g., an excitatory CS-shock association). Extinction in Phase 2 does not destroy the memory corresponding to Phase 1; instead, presenting the CS without shock results in the storage of a second, conflicting memory involving the CS (e.g., a CS-no-shock, or inhibitory CS-shock, association; Konorski, 1967; Pearce, 1987; Pearce \& Hall, 1980; Wagner, 1981). Memories corresponding to each phase are thus stored and available following extinction (e.g., Bouton, 1993). Performance after Phase 2 therefore hinges on which memory is retrieved. The memories corresponding to each phase depend differentially on context for retrieval. In extinction, the Phase 2 (CS-no-shock, or shock-inhibition) memory is more dependent on context for retrieval than the Phase 1 (CS-shock) memory. As a consequence, a switch out of the Phase 2 context may reduce retrieval of the $\mathrm{CS}$-no-shock memory more than retrieval of CS-shock (Bouton, 1993).

This account views counterconditioning as the same as extinction, except, of course, that the CS is associated with a different US (e.g., Bouton, 1993; Bouton \& Peck, 1992). An excitatory CS-shock association is stored during Phase 1. CS-food pairings in Phase 2 promote the storage of two new associations involving the CS. One is a CS-no-shock (an inhibitory CS-shock) association, just as in extinction. The other is a second, excitatory association involving the new US (e.g., CS-food). As in extinction, the memories corresponding to each phase are stored and available following Phase 2 , and memory retrieval again depends differentially on context. A switch out of the Phase 2 context may reduce retrieval of the Phase 2 CS-no-shock memory more than the Phase 1 CS-shock memory. Relatively stronger retrieval of CS-shock may then interfere with retrieval of the Phase 2 CS-food memory (see Bouton, 1993; Bouton \& Peck, 1992).

This view can account for spontaneous recovery, renewal, and reinstatement by emphasizing the effects of context on retrieval of different memories. Spontaneous recovery results when time passes after Phase 2; since the passage of time is viewed as a gradually changing context, testing takes place in a temporal context different from that of Phase 2. Renewal results when the Phase 2 apparatus cues are replaced by Phase 1 cues or by different cues. Reinstatement results because US exposure conditions excitation to the context. Contextual excitation may make the test context more similar to the context associated with Phase 1 (Bouton, 1993). It may also simply reduce the similarity between the testing and Phase 2 contexts, which in itself can cause a renewal effect (Bouton \& Ricker, 1994). (Previous research argues against the idea that contextual excitation merely summates with excitation to the CS; see Bouton, 1984; Bouton \& King, 1986; Bouton, Rosengard, Achenbach, Peck, \& Brooks, 1993). A memory retrieval view (Bouton, 1991, 1993) can integrate several response-recovery effects that occur after both extinction and counterconditioning.

\section{REFERENCES}

Archer, T., S.ödén, P.-O., Nilsson, L.-G., \& Car'TER, N. (1979). Role of exteroceptive background context in taste-aversion conditioning and extinction. Animal Learning \& Behavior, 7, 17-22.

Baker, A. G., Steinwald, H., \& Bouton, M. E. (1991). Contextual conditioning and reinstatement of extinguished instrumental responding. Quarterly Journal of Experimental Psychology, 43B, 199-218.

Bouton, M. E. (1984). Differential control by context in the inflation and reinstatement paradigms. Journal of Experimental Psychology: Animal Behavior Processes, 10, 56-74.

Bouton, M. E. (1991). Context and retrieval in extinction and in other examples of interference in simple associative learning. In L. Dachowski \& C. F. Flaherty (Eds.), Current topics in animal learning: Brain, emotion and cognition (pp. 25-53). Hillsdale, NJ: Erlbaum. Bouton, M. E. (1993). Context, time, and memory retrieval in the interference paradigms of Pavlovian learning. Psychological Bulletin, $114,80-99$.

Bouton, M. E., \& Bolles, R. C. (1979). Rule of conditioned contextual stimuli in reinstatement of extinguished fear. Journal of Experimental Psychology: Animal Behavior Processes, 5, 368-378.

Bouton, M. E., \& KING, D. A. (1983). Contextual control of the extinction of conditioned fear: Tests for the associative value of the context. Journal of Experimental Psychology: Animal Behavior Processes, 9, 248-265.

Bouton, M. E., \& King, D. A. (1986). Effect of context on performance to conditioned stimuli with mixed histories of reinforcement and nonreinforcement. Journal of Experimental Psychology: Animal Behavior Processes, 12, 4-15.

Bouton, M. E., \& Peck, C. A. (1989). Context effects on conditioning, extinction, and reinstatement in an appetitive conditioning preparation. Animal Learning \& Behavior, 17, 188-198.

Bouton, M. E., \& PECK, C. A. (1992). Spontaneous recovery in cross- 
motivational transfer (counterconditioning). Animal Learning \& Behavior, 20, 313-321.

Bouton, M. E., \& Ricker, S. T. (1994). Renewal of extinguished responding in a second context. Animal Learning \& Behavior, 22, 317 . 324.

Bouton, M. E., Rosengard, C., Achenbach, G., Peck, C. A., \& BrooKs, D. C. (1993). Effects of contextual conditioning and unconditional stimulus presentation on performance in appetitive conditioning. Quarterly Journal of Experimental Psychology, 46B, 63-95.

Bouton, M. E., \& Swartzentruber, D. (1989). Slow reacquisition following extinction: Context, encoding, and retrieval mechanisms. Journal of Experimental Psychology: Animal Behavior Processes, 15, 43-53.

Bromage, B. K., \& SCAvio, M. J., JR. (1978). Effects of an aversive CS + and $\mathrm{CS}-$ under deprivation upon successive classical appetitive and aversive conditioning. Animal Learning \& Behavior, 6, 57-65.

Brooks, D. C., \& Bouton, M. E. (1993). A retrieval cue for extinction attenuates spontaneous recovery. Journal of Experimental Psychology: Animal Behavior Processes, 19, 77-89.

Brooks, D. C., \& Bouton, M. E. (1994). A retrieval cue for extinction attenuates response recovery (renewal) caused by a return to the conditioning context. Journal of Experimental Psychology: Animal Behavior Processes, 20, 366-379.

Howell, D. C. (1987). Statistical methods for psychology (2nd ed.). Boston: PWS-Kent.

Kaye, H., Preston, G. C., Szabo, L., Druiff, H., \& Mackintosh, N. J. (1987). Context specificity of conditioning and latent inhibition: Evidence for a dissociation of latent inhibition and associative interference. Quarterly Journal of Experimental Psychology, 39B, 127-145.

KONORSKI, J. (1967). Integrative activity of the brain. Chicago: University of Chicago Press.

PAvLov, I. P. (1927). Conditioned reflexes: An investigation of the phys- iological activity of the cerebral cortex (G. V. Anrep, Ed. and Trans.). London: Oxford University Press.

PEARCE, J. M. (1987). A model for stimulus generalization in Pavlovian conditioning. Psychological Review, 94, 61-73.

Pearce, J. M., \& Hall, G. (1980). A model for Pavlovian learning: Variations in the effectiveness of conditioned but not of unconditioned stimuli. Psychological Review, 87, 532-552.

Peck, C. A., \& Bouton, M. E. (1990). Context and performance in aversive-to-appetitive and appetitive-to-aversive transfer. Learning \& Motivation, 21, 1-21.

Rescorla, R. A., \& CUnNingham, C. L. (1978). Recovery of the US representation over time during extinction. Learning \& Motivation, 9 , 373-391.

Rescorla, R. A., \& Heth, C. D. (1975). Reinstatement of fear to an extinguished conditioned stimulus. Journal of Experimental Psychology: Animal Behavior Processes, 1, 88-96.

RoBbins, S. J. (1990). Mechanisms underlying spontaneous recovery in autoshaping. Journal of Experimental Psychology: Animal Behavior Processes, 16, 235-249.

SCAvio, M. J. (1974). Classical-classical transfer: Effects of prior aversive conditioning upon appetitive conditioning in rabbits. Journal of Comparative \& Physiological Psychology, 86, 107-115.

Thomas, D. R., \& Sherman, L. (1986). An assessment of the role of handling cues in "spontaneous recovery" after extinction. Journal of the Experimental Analysis of Behavior, 46, 305-314.

WAGNER, A. R. (1981). SOP: A model of automatic memory processing in animal behavior. In N. E. Spear \& R. R. Miller (Eds.), Information processing in animals: Memory mechanisms (pp. 5-47). Hillsdale. NJ: Erlbaum.

(Manuscript received January 24, 1994; revision accepted for publication November 3, 1994.) 